\title{
ESTIMATIONS OF HERON MEANS FOR POSITIVE OPERATORS
}

\section{Masatoshi Fuji, Shigeru Furuichi AND Ritsuo NAKAMOTO}

Abstract. The arithmetic-geometric mean inequality induces the path of Heron means through these two means by $H_{r}^{\mu}(A, B)=r\left(A \sharp_{\mu} B\right)+(1-r)\left(A \nabla_{\mu} B\right)$ for each $\mu \in[0,1], r \in \mathbb{R}$ and positive operators $A, B$ on a Hilbert space. In this note, we estimate $H_{r}^{\mu}(A, B)$ by the harmonic mean. As an application of this method, we refine the arithmetic-geometric mean inequality under the assumption of the strict order $A-B \geqslant m>0$.

Mathematics subject classification (2010): 47A63.

Keywords and phrases: Arithmetic mean, geometric mean, arithmetic-geometric mean inequality and Heron mean.

\section{REFERENCES}

[1] R. BHATIA, Interpolating the arithmetic-geometric mean inequality and its operator version, Linear Algebra Appl., 413 (2006), 355-363.

[2] M. FUJII, Y. O. Kim AND R. NAKAMOTO, A characterization of convex functions and its application to operator monotone functions, Banach J. Math. Anal., 8 (2014), 118-123.

[3] M. Fujil, M. S. Moslehian, H. Najafi And R. NAKAmoto, Estimates of operator convex and operator monotone functions on bounded intervals, Hokkaido Math. J., to appear.

[4] S. FURUICHI, Operator inequalities among arithmetic mean, geometric mean and harmonic mean, J. Math. Inequal., 8 (2014), 669-672. 